\title{
A GENERALIZED PRESSING-DOWN LEMMA AND ISOCOMPACTNESS
}

\author{
BRIAN M. SCOTT
}

\begin{abstract}
A topological generalization of the Pressing-Down Lemma ( Neumer's Theorem) is formulated and proved. It is shown that this result implies several recent results on isocompactness due to Worrell and Wicke.
\end{abstract}

Introduction. Let $\kappa$ be an uncountable regular cardinal. A set $K \subseteq \kappa$ is a $c u b$ ( $=$ closed unbounded) set in $\kappa$ if $K$ is cofinal in $\kappa$ and closed in the order topology on $\kappa$. A set $S \subseteq \kappa$ is stationary in $\kappa$ iff $S \cap K \neq \varnothing$ for every cub set $K$ in $\kappa$. If $A \subseteq \kappa$, a function $f: A \rightarrow \kappa$ is a pressing-down (or regressive) function iff $f(\alpha)<\alpha$ for each $\alpha \in A$. One of the most useful elementary results in set theory is the

Pressing-Down Lemma. If $S$ is a stationary subset of the uncountable regular cardinal $\kappa$, and $f: S \rightarrow \kappa$ is a pressing-down function, then there are an $\eta \in \kappa$ and $a$ $C \in[S]^{\kappa}$ such that $f(\alpha)<\eta$ for all $\alpha \in C .\left([S]^{\kappa}=\{A \subseteq S:|A|=\kappa\}\right.$.)

In this form the Pressing-Down Lemma (PDL) is also known as Neumer's Theorem [Ne]. A stronger version, $\mathrm{PDL}^{+}$, is due to Fodor [Fo]; it asserts that the set $C$ can be chosen to be a stationary set. (Actually, both the PDL and the PDL ${ }^{+}$ are frequently stated in a slightly different form in which ' $f(\alpha)<\eta$ ' is replaced by ' $f(\alpha)=\eta$ '. This is only an apparent strengthening, since the union of fewer than $\kappa$ bounded (nonstationary, resp.) subsets of $\kappa$ is bounded (nonstationary, resp.), and the present formulation is more apt for the sequel.)

By generalizing the notion of a cofinal set, Juhász [Ju] has found one purely topological generalization of the PDL. In this note I generalize the notion of a cub set to give another topological version of the PDL, one which implies some recent results of Worrell and Wicke on isocompactness and weak covering properties [WoWi $\mathbf{W}_{2}$ ]. (However, the proof of (i) of the Main Theorem below is very similar to the argument in [Ju].)

Definitions. Let $X$ be a topological space. If $\mathscr{F}$ is a free closed filter on $X$, let $k(\mathscr{F})=\inf \left\{\kappa \geqslant \omega: \exists \mathscr{Q} \in[\mathscr{F}]^{\kappa}(\cap \mathbb{Q}=\varnothing)\right\}$, and let $\overline{\mathscr{F}}=\left\{\cap \mathbb{Q}: \mathbb{Q} \in[\mathscr{F}]^{<k(\mathscr{F})}\right\}$. (As usual, $[A]^{<\kappa}$ denotes $\{B \subseteq A:|B|<\kappa\}$.) The following observations are easily verified.

(1) $\overline{\mathscr{F}}$ is a free closed filter on $X$.

(2) $k(\overline{\mathscr{F}})=\operatorname{cf} k(\mathscr{F})$.

Received by the editors April 26, 1979 and, in revised form, March 25, 1980.

AMS (MOS) subject classifications (1970). Primary 54A25; Secondary 54D20, 02A20.

Key words and phrases. Pressing-Down Lemma, Neumer's Theorem, closed filter, isocompactness. 
(3) $\overline{\mathscr{F}}$ is $k(\overline{\mathscr{F}})$-complete (= closed under intersections of fewer than $k(\overline{\mathscr{F}})$ of its elements).

(4) $\overline{\overline{\mathscr{F}}}=\overline{\mathscr{F}}$.

Say that $\mathscr{F}$ is regular iff $\mathscr{F}=\overline{\mathscr{F}}$.

(5) Every free closed ultrafilter on $X$ is regular.

(6) If $\mathscr{F}$ is regular, then $k(\mathscr{F})$ is a regular cardinal. (For cf $k(\mathscr{F})=k(\bar{F})=k(\mathscr{F})$.)

(7) If $\kappa>\omega$ is a regular cardinal, then $\mathscr{F}=\{K \subseteq \kappa: K$ is a cub set in $\kappa\}$ is a (regular) free closed ultrafilter on $\kappa$, and $k(\mathscr{F})=\kappa$.

Observation (7) provides a basis for generalizing the PDL.

Now fix a regular free closed filter, $\mathscr{F}$, on $X$. A set $S \subseteq X$ is $\mathscr{F}$-stationary iff $\forall F \in \mathscr{F}(S \cap F \neq \varnothing)$; otherwise, $S$ is $\mathscr{F}$-nonstationary. $\mathscr{F}^{*}=\{A \subseteq X: \exists F \in \mathscr{F}$ $(F \subseteq A)\}$, the filter (on $\mathscr{P}(X)$ ) generated by $\mathscr{F}$. A neighbornet on a set $A \subseteq X$ is a function $V: A \rightarrow \tau X$ (= the topology on $X$ ) such that $x \in V(x)$ for all $x \in A ; V$ is $\mathscr{F}$-bounded iff $V(x)$ is $\mathscr{F}$-nonstationary for all $x \in A$, and $V$ is pseudoconstant at $x$ on $C$ (where $C \subseteq A$ ) iff $x \in \cap V[C]$.

(8) If $\kappa>\omega$ is a regular cardinal, $\mathscr{F}=\{K \subseteq \kappa: K$ is a cub set in $\kappa\}, A \subseteq \kappa$, and $f: A \rightarrow \kappa$ is a pressing-down function, then the neighbornet $V: A \rightarrow \tau \kappa: \alpha \mapsto(f(\alpha)$, $\alpha]$ is $\mathscr{F}$-bounded.

Finally, $\Delta^{*}(X)=\inf \{\kappa \geqslant \omega: X$ has no closed, discrete subset of power $\kappa\}$, and $s^{*}(X)=\inf \{\kappa>\omega: X$ has no (relatively) discrete subset of power $\kappa\}$; obviously $\Delta(X)<\Delta^{*}(X)<s^{*}(X) \geqslant s^{*}(X)$, where $s(X)$ and $\Delta(X)$ are, respectively, the spread and the closed discreteness character of $X$. And if $x \in X$ and $\mathcal{C} \subseteq \mathcal{P}(X)$, then $\operatorname{st}(x, \mathcal{C})=\cup\{C \in \mathcal{C}: x \in C\}$.

Results. The following propositions have straightforward proofs.

Proposition 0. If $S \subseteq X$ is $\mathscr{F}$-stationary, where $\mathscr{F}$ is a regular free closed filter on $X$, then $S$ is not the union of fewer than $k(\mathcal{F}) \mathscr{F}$-nonstationary subsets of $X$.

Proposition 1. If $W \in \tau X$ is $\mathscr{F}$-stationary, where $\mathscr{F}$ is a free closed ultrafilter on $X$, then $W \in \mathscr{F}^{*}$.

MAIN Theorem. Let $S \subseteq X$ be $\mathcal{F}$-stationary, where $\mathcal{F}$ is a regular free closed filter on $X$, and let $V: S \rightarrow \tau X$ be an $\mathscr{F}$-bounded neighbornet. Assume further that $k(\mathcal{F})>\Delta^{*}(X)$.

(i) If $k(\mathscr{F})>s^{*}(X)$, or $\mathscr{F}$ is a closed ultrafilter, then there is an $x \in S$ such that

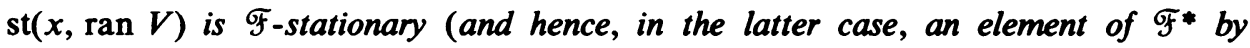
Proposition 1).

(ii) If $k(\mathscr{F}) \geqslant s^{*}(X)$, or $\mathscr{F}$ is a closed ultrafilter, then there are an $x \in S$ and $a$ $C \in[S]^{k(\mathcal{F})}$ such that $V$ is pseudoconstant at $x$ on $C$.

(iii) If $k(\mathcal{F})>s^{*}(X)$, then there are an $x \in S$ and an $\mathscr{F}$-stationary $T \subseteq S$ such that $V$ is pseudoconstant at $x$ on $T$.

$\Delta^{*}(\kappa)=\omega$ for any regular cardinal $\kappa>\omega$, so the PDL is an immediate consequence of (ii) and observations (7) and (8).

Proof of Main Theorem. Note first that (i) implies (ii). Let $x$ be as in (i), so that st $(x, \operatorname{ran} V)$ is $\mathscr{F}$-stationary, and let $C=\{y \in S: x \in V(y)\}$. Let $W=\cup V[C]$; 


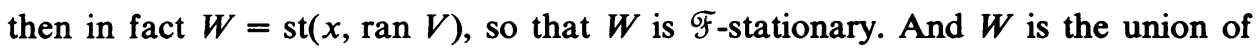
$|C| \mathscr{F}$-nonstationary sets, so-by Proposition $0-|C|>k(\mathscr{F})$.

We now prove (i). Let $W=\cup$ ran $V$, so that $S \subseteq W \in \tau X$. If $\mathscr{F}$ is a closed ultrafilter, then by Proposition $1, W \in \mathscr{F}^{*}$, so there is an $F_{0} \in \mathscr{F}$ such that $F_{0} \subseteq W$; let $A=S \cap F_{0}$. Otherwise, let $A=S$. (In either case $A$ is $\mathscr{F}$-stationary.) Let $D \subseteq A$ be maximal with respect to the following property: if $x, y \in D$, and $x \neq y$, then $x \notin \operatorname{st}(y, \operatorname{ran} V)$. Clearly $D$ is discrete, so $|D|<s^{*}(X)$. Moreover, $D$ is closed in $W$; thus, if $\mathscr{F}$ is a closed ultrafilter, then $D$ is closed in $F_{0}$ (and hence in $X)$, so in this case we even have that $|D|<\Delta^{*}(X)$. In either case, $|D|<k(\mathscr{F})$. But $\operatorname{st}(D, \operatorname{ran} V)(=\cup\{\operatorname{st}(x, \operatorname{ran} V): x \in D\})$, since it contains $A$ (by the maximality of $D)$, is $\mathcal{F}$-stationary, so, by Proposition $0, \operatorname{st}(x, \operatorname{ran} V)$ is $\mathcal{F}$-stationary for some $x \in D$.

To prove (iii), suppose that $V$ is not pseudoconstant on any $\mathscr{F}$-stationary $T \subseteq S$, and let $\lambda=k(\mathscr{F})$. Suppose further that $\alpha<\lambda$ and that we have already chosen points $x_{\xi} \in S$ (for $\xi<\alpha$ ) such that for each $\eta<\alpha,\left\{x_{\xi}: \xi<\alpha\right\} \cap V\left(x_{\eta}\right)=\left\{x_{\eta}\right\}$. Let $A=\left\{x_{\xi}: \xi<\alpha\right\} ;|A|<\lambda$, so $V[A]$ is $\mathscr{F}$-nonstationary. Fix $F_{0} \in \mathscr{F}$ disjoint from $V[A]$. Moreover, $\mathscr{F}$ is $\lambda$-complete, and $V$ is not pseudoconstant on an $\mathscr{F}$-stationary $T \subseteq S$ at any $x \in A$, so there is an $F_{1} \in \mathcal{F}$ such that $F_{1} \subseteq F_{0}$ and $V\left[F_{1} \cap S\right] \cap A=\varnothing$. Pick $x_{\alpha} \in F_{1} \cap S$. Then $x_{\alpha} \notin V[A]$, and $V\left(x_{\alpha}\right) \cap A=\varnothing$.

Thus, the recursion goes through to $\lambda$, and there is a $D_{0} \in[S]^{\lambda}$ such that $V(x) \cap D_{0}=\{x\}$ for each $x \in D_{0}$. Clearly $D_{0}$ is discrete, which is impossible, since $s^{*}(X) \leqslant \lambda=\left|D_{0}\right|$. This completes the proof.

Part (iii) of the theorem is the best approximation I could get to the $\mathrm{PDL}^{+}$, but it does not imply the $\mathrm{PDL}^{+}$, since $s^{*}(\kappa)=\kappa^{+}$for any cardinal $\kappa$. (Juhász was also unable to extend his generalization to include the $\mathrm{PDL}^{+}$.) I suspect that the difficulty is inherent in the nature of the $\mathrm{PDL}^{+}$, which seems to require some notion of diagonal intersection for its proof: diagonal intersections are heavily dependent upon considerations of order and cardinality.

An application. A popular question in recent years has been: How weak can a covering property, $P$, be and still satisfy the equation "countably compact $+P=$ compact'? The excellent solution ' $\boldsymbol{P}=$ weakly $\boldsymbol{\delta} \theta$-refinable' [WiWo] is notorious for having been overlooked for ten years after its first mention in [WoWi $\mathbf{i}_{1}$. Even it, however, is superseded by the results in [ $\left.\mathbf{W o W i}_{2}\right]$, of which it is an easy corollary. Here I show that (a strengthening of) the main theorem of [ $\left.\mathbf{W o W i}_{2}\right]$ is itself a corollary of the above generalized PDL.

If $X$ is a space, $\kappa$ is a cardinal, and $\mathscr{U}$ is an open cover of $X$, a weak [k]-refinement of $\mathscr{U}$ is an open refinement, $\mathscr{R}=U\left\{\mathscr{R}_{\alpha}: \alpha \in \lambda\right\}$, of $\mathscr{U}$ such that: (a) $\lambda<\kappa$, and (b) for each $x \in X$ there is an $\alpha \in \lambda$ such that $0<\mid\left\{R \in \Re_{\alpha}\right.$ : $x \in R\} \mid<\kappa$. The main theorem of [ $\mathbf{W o W i}_{2}$ ] (slightly modified) can now be stated as follows.

COROllaRY 1 [ $\mathrm{WoWi}_{2}$ ]. Let $X$ be a $T_{1}$-space such that every well-ordered, increasing open cover, $Q$, of $X$ of regular, uncountable cardinality has a weak $[|थ|]-$ refinement. If $\mathscr{F}$ is a free closed ultrafilter on $X$, then $k(\mathcal{F})<\Delta^{*}(X)$. 
In fact, however, a stronger result is true. In the definition given above of a weak [ $\kappa$ ]-refinement of $\mathcal{Q}$, replace condition (b) by the following weaker condition $\left(\mathrm{b}^{\prime}\right)$ : for each $x \in X$ there are an $\alpha \in \kappa$ and a $\mathscr{V} \in[\mathcal{Q}]^{<\kappa}$ such that $\varnothing \neq \operatorname{st}\left(x, \mathscr{R}_{\alpha}\right) \subseteq$ $\cup \mathcal{V}$. An $R$ satisfying (a) and $\left(b^{\prime}\right)$ will be called a very weak $[\kappa]$-refinement of $\mathcal{Q}$.

COROLlaRY 1'. Let $X$ be a $T_{1}$-space such that every well-ordered, increasing open cover, $\mathcal{Q}$, of $X$ of regular uncountable cardinality has a very weak $[|\mathcal{U}|]-$-refinement. If $\mathcal{F}$ is a free closed ultrafilter on $X$, then $k(\mathcal{F})<\Delta^{*}(X)$.

Proof. Suppose that $\mathscr{F}$ is a free closed ultrafilter on $X$ for which $k(\mathscr{F})>\Delta^{*}(X)$, and let $\lambda=k(\mathscr{F})$. Clearly $\lambda>\omega$ unless $\lambda=\Delta^{*}(X)=\omega$; but $\Delta^{*}(X)=\omega$ iff $X$ is countably compact, in which case $\mathcal{F}$ is at least $\omega_{1}$-complete, so in all cases $\lambda>\omega$. And of course $\lambda$ is regular.

Let $\mathscr{F}_{0}=\left\{F_{\alpha}: \alpha \in \lambda\right\} \subseteq \mathscr{F}$ be such that $\cap \mathscr{F}_{0}=\varnothing$ and $F_{\alpha} \supset F_{\beta}$ whenever $\alpha<\beta<\lambda$, and, for $\alpha \in \lambda$, let $U_{\alpha}=X \backslash F_{\alpha}$. Let $\mathscr{R}=\cup\left\{\mathscr{R}_{\alpha}: \alpha \in \kappa\right\}$ be a very weak $[\lambda]$-refinement of $\left\{U_{\alpha}: \alpha \in \lambda\right\}$ (as described above) for some $\kappa<\lambda$. For each $\alpha \in \kappa$, let $A_{\alpha}=\left\{x \in X: \varnothing \neq \operatorname{st}\left(x, \Re_{\alpha}\right) \subseteq \cup\left\{U_{\alpha}: \alpha<\mu\right\}\right.$ for some $\left.\mu<\lambda\right\}$. Then $X=\cup\left\{A_{\alpha}: \alpha \in \kappa\right\}$, so, by Proposition 0 , there is an $\eta \in \kappa$ such that $A_{\eta}$ is $\mathscr{F}$-stationary. Let $N: A_{\eta} \rightarrow \Re_{\eta}$ be a neighbornet; there is then, by the Main Theorem, an $x \in A_{\eta}$ for which $\operatorname{st}(x, \operatorname{ran} N)$ is $\mathscr{F}$-stationary. But then $\operatorname{st}(x, \operatorname{ran} N)$ $\subseteq \operatorname{st}\left(x, \Re_{\alpha}\right) \subseteq U\left\{U_{\alpha}: \alpha<\mu\right\}$ for some $\mu<\lambda$, so, again by Proposition 0 , some $U_{\alpha}(\alpha<\mu)$ is $\mathscr{F}$-stationary-which is absurd. Thus, no such $\mathscr{F}$ exists.

A similar argument yields the following results, given the observation that $s^{*}(X)>\omega$ for any infinite Hausdorff space $X$.

COROllary 2. Let $X$ be as in Corollary $1^{\prime}$, but assume further that $X$ is an infinite Hausdorff space. If $\mathscr{F}$ is a regular free closed filter on $X$, then $k(\mathscr{F})<s^{*}(X)$.

Corollary 3. Let $X$ be as in Corollary 2, and let $\mathcal{F}$ be a free closed filter on $X$. Then $\operatorname{cf}(\mathscr{F})<s^{*}(X)$.

Proof. Apply Corollary 2 to $\overline{\mathscr{F}}$.

REMARK. No separation axioms are required in the above corollaries if the conclusions are weakened to ' $k(\mathscr{F}) \leqslant \Delta(X)$ ', ' $k(\mathscr{F}) \leqslant s(X)$ ', and 'cf $k(\mathscr{F})<s(X)$ ', respectively. Moreover, Corollaries 2 and 3 almost apply to $T_{1}$-spaces. First, it is clearly sufficient to assume that $X$ has an infinite Hausdorff subspace, for that is equivalent to $s^{*}(X)>\omega$. And it can be shown fairly easily that a $T_{1}$-space has no infinite Hausdorff subspace iff it is hereditarily compact, in which case the magnitude of $k(\mathscr{F})$ is unlikely to be of much interest.

Corollary 4. If $X$ is as in Corollary $1^{\prime}$, then $X$ is linearly $\left(\infty, s(X)^{+}\right)$-compact, i.e., every linearly ordered (by inclusion) open cover of $X$ has a subcover of cardinality at most $s(X)$. 


\section{REFERENCES}

[Fo] G. Fodor, Eine Bemerkung zur Theorie der regressiven Funktionen, Acta Sci. Math. (Szeged) 17 (1956), 139-142.

[Ju] I. Juhász, On Neumer's Theorem, Proc. Amer. Math. Soc. 54 (1976), 453-454.

[Ne] W. Neumer, Verallgemeinerung eines Satzes von Alexandroff und Unysohn, Math. Z. 54 (1951), 254-261.

[WiWo] H. H. Wicke and J. M. Worrell, Jr., Point-countability and compactness, Proc. Amer. Math. Soc. 55 (1976), 427-431.

[WoWi ] J. M. Worrell, Jr. and H. H. Wicke, Characterizations of developable topological spaces, Canad. J. Math. 17 (1965), 820-830.

[WoWi $]$ _ A covering property which implies isocompactness. I (preprint).

Department of Mathematics, Cleveland State University, Cleveland, Ohio 44115 